\title{
Fuzzy Logic Based Multiplexer Design \& Simulation
}

\author{
Furqan Fazili \\ Department of Electronics \& Communication Engineering, Islamic University of Science \& Technology, \\ Awantipora, India
}

Abstract: In this paper we try to design and implement the logic of a Fuzzy Multiplexer (fMUX) system. A design of Fuzzy Multiplexer is presented and its generalization to classical logic has been shown with simulations. In fuzzy modelling, a fuzzy multiplexer fMUX acts as a fuzzy switch to select one of the fuzzy inputs. Selection of the inputs is determined by the value of candidate inputs termed as "Select Inputs". These fMUX's could be used as generic building modules in fuzzy systems. All simulations were done using MultiSim 11.0 .

Keywords: Fuzzy logic, Fuzzy Gates, Fuzzy Multiplexer, Selection Lines, Fuzzy Generalization, Simulation.

\section{Introduction}

Fuzzy logic is a form of many-valued logic; it deals with reasoning that is approximate rather than fixed and exact. Compared to traditional binary sets, where variables may take on true or false values, fuzzy logic variables may have a truth value that ranges in degree between 0 and 1. Fuzzy logic has been extended to handle the concept of partial truth, where the truth value may range between completely true and completely false. The main characteristic of the fuzzy logic is the use of continuous, rather than discrete, waveforms.

\section{Fuzzy Logic Gates}

In order to develop a multiplexer based on fuzzy logic we would need fuzzy logic gates to perform fuzzy logic operation of AND,NOT and OR. The designs presented in [1] are used in this paper, however during implementation of these designs, accurate results were not obtained, so certain small modification to the design was introduced which are shown in next section. This modification does not imply any fault in their design, but was needed to for proper simulation.

For the fuzzy logic "P", "Q," ... will have values in the closed interval $\{0,1\}$, and "P", "Q",.. will be the value of the propositional variable.

\section{A. Fuzzy AND}

The Fuzzy AND or F-AND is shown in Fig 1.

The fuzzy gate "AND" has the following definition in

fuzzy logics:

$$
|\mathrm{P} \wedge \mathrm{Q}|=\min (\mathrm{P}, \mathrm{Q})
$$

Results are show in Fig 2 and Fig 3.

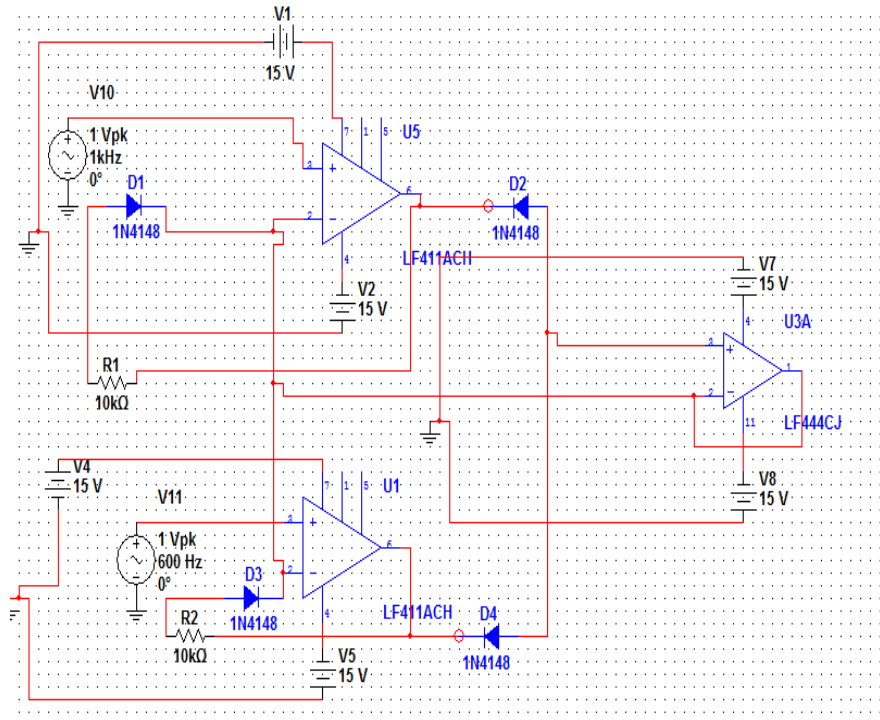

Figure 1: Circuit Diagram of F-AND 


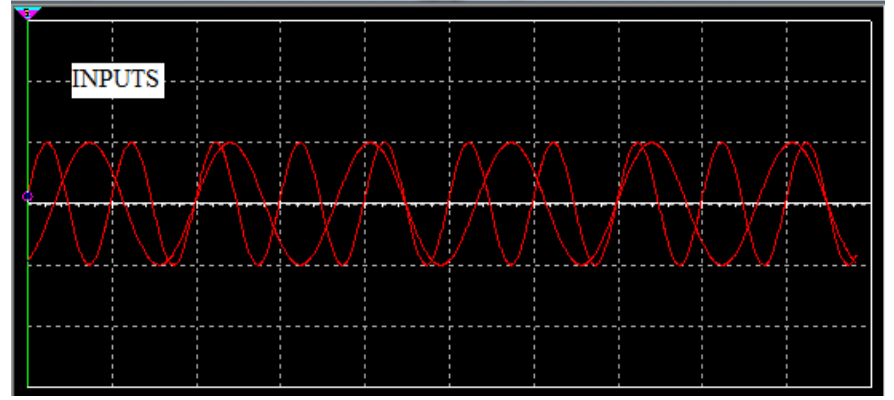

Figure 2: Input waveforms of F-AND

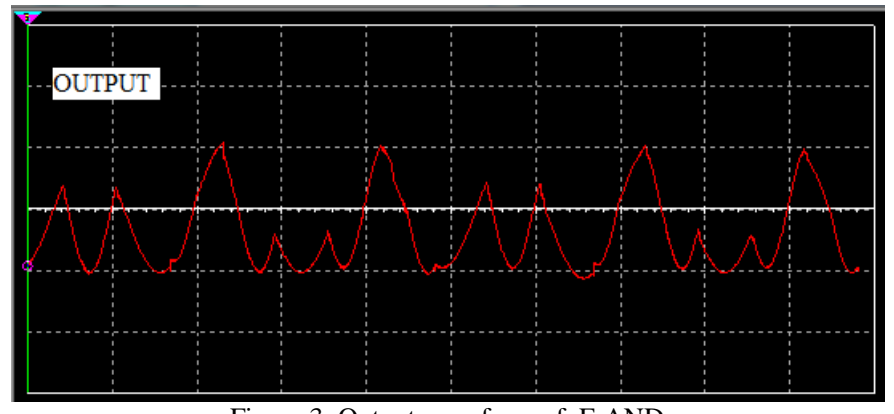

B. Fuzzy OR

Figure 3: Output waveform of F-AND

The Fuzzy OR or F-OR is shown in Fig 4

The fuzzy gate "or" has the following definition in

fuzzy logics:

$$
|\mathrm{P} \vee \mathrm{Q}|=\max (\mathrm{P}, \mathrm{Q})
$$

Its results are show in Fig 5 and Fig 6

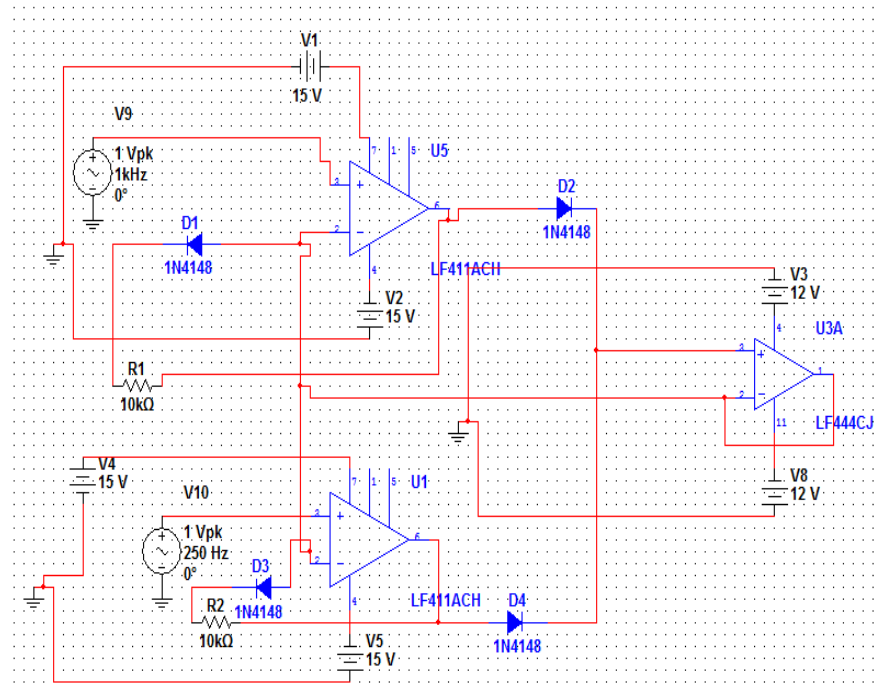

Figure. 4: Circuit Diagram of F-OR

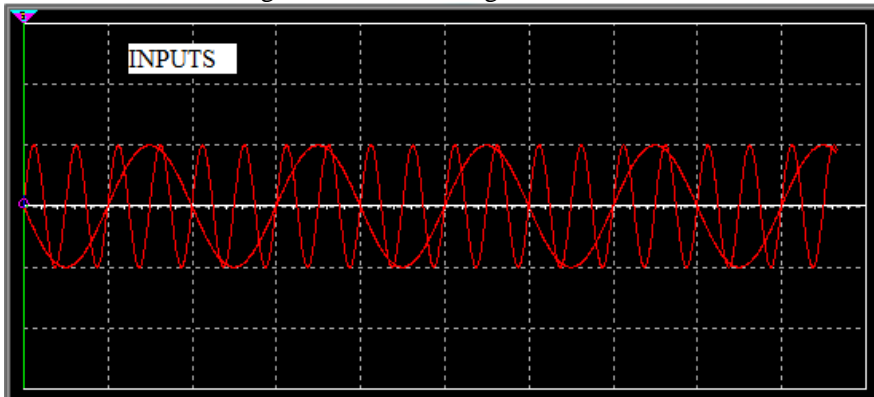

Figure 5: Input waveforms of F-OR 


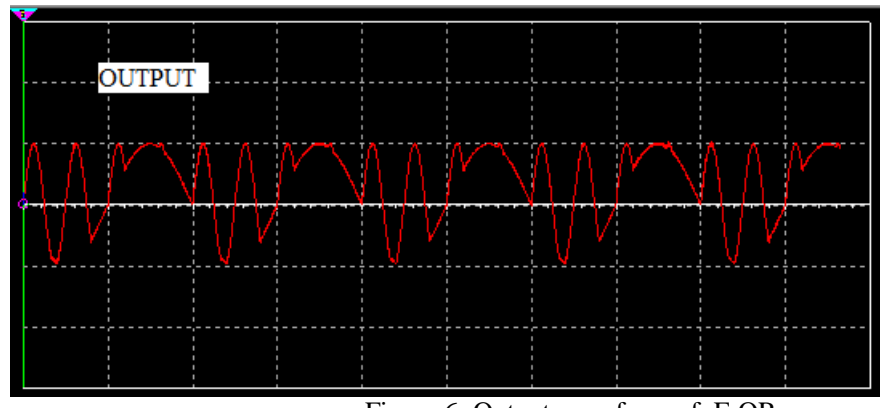

Figure 6: Output waveform of F-OR

C. Fuzzy NOT

The Fuzzy Not or F-NOT is shown in Fig 7.

The fuzzy gate "not" has the following definition in

fuzzy logics:

$$
\neg \mathrm{P}=1-|\mathrm{P}|
$$

Its results are show in Fig 8 and Fig 9

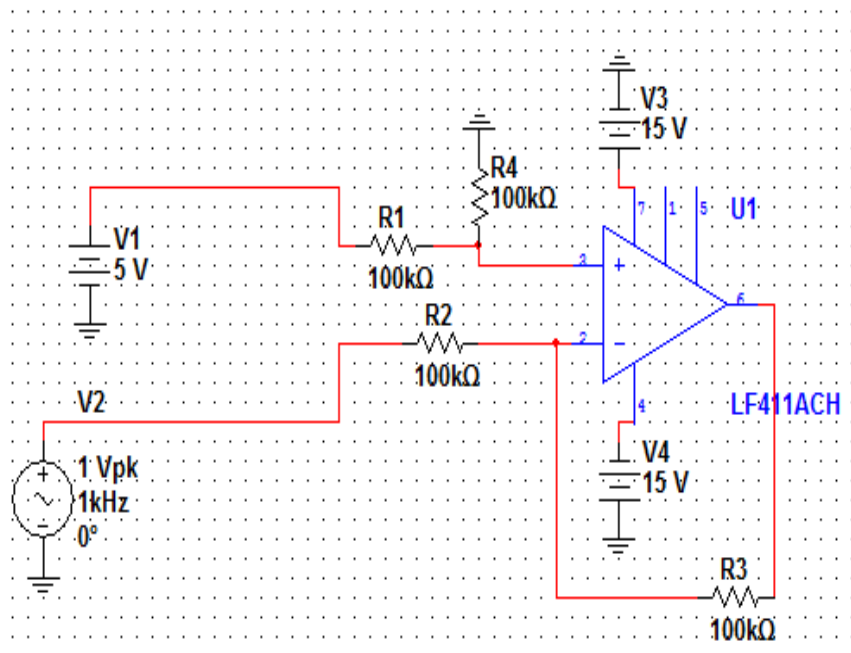

Figure 7: Circuit Diagram of F-NOT

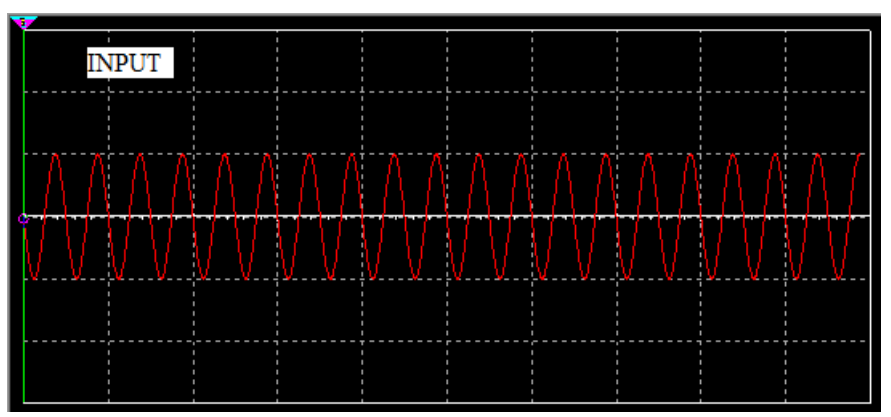

Figure: 8 Input waveform of F-NOT

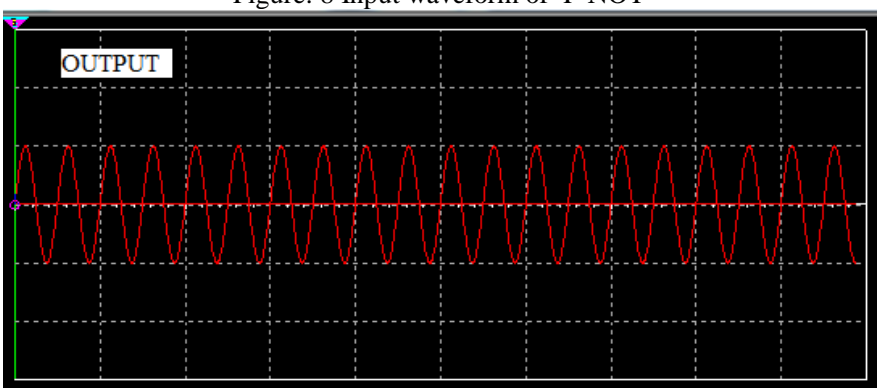

Figure 9: Output waveform of F-NOT 


\section{Design Of Fmux}

A multiplexer (or MUX) is a device that selects one of several analog or digital input signals and forwards the selected input into a single line using selection logic provided by select lines.

The basic fuzzy multiplexer, fMUX, realizes fuzzy multiplexing by the general expression [3]

$$
y=P * \bar{x}+Q * x
$$

where the logic operations ( $*$ and + ) are implemented using some t- and s-norms. The fMUX can be represented in a block diagram as follows

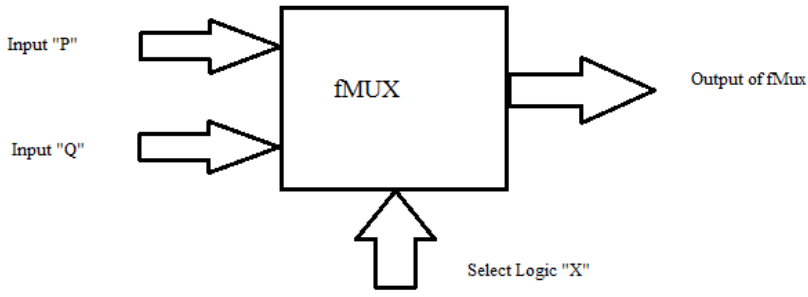

Figure 10 : fMUX Block Diagram

The fMUX is given inputs " $\mathrm{P}$ " and " $\mathrm{Q}$ " and the output is generated depending on the select logic " $\mathrm{X}$ ". The variable (x) in the above expression plays the role of a switching variable (selection or select), that allows two fixed information inputs ( $\mathrm{P}$ or $\mathrm{Q}$ ) to affect the output. The degree to which the produced result depends on these fixed information values is controlled by the select variable.

The electronic circuit which realizes the above expression is shown if Fig. 11. The input "P" is connected to 5V, $1 \mathrm{kHz}$ AC Voltage source while as input "Q" is connected to $5 \mathrm{~V}, 2 \mathrm{kHz}$ Ac voltage source, and the selection logic line " $\mathrm{X}$ " is connected to $5 \mathrm{~V}, 3 \mathrm{kHz}$ AC Voltage source.

The design of F-AND , F-NOT and F-OR fuzzy logic gates utilized in the circuit has been already described and simulations for individual gates have already been shown. The simulation for fMUX was done using MultisSim and the results were as follows in time base of $500 \mathrm{us} / \mathrm{Div}$ and voltage scale of 5V/Div.

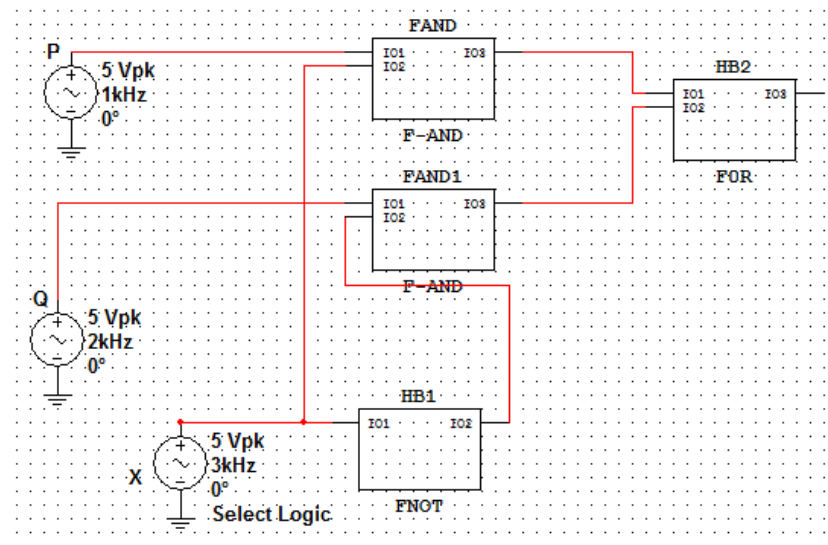

Figure 11. fMUX Circuit Diagram

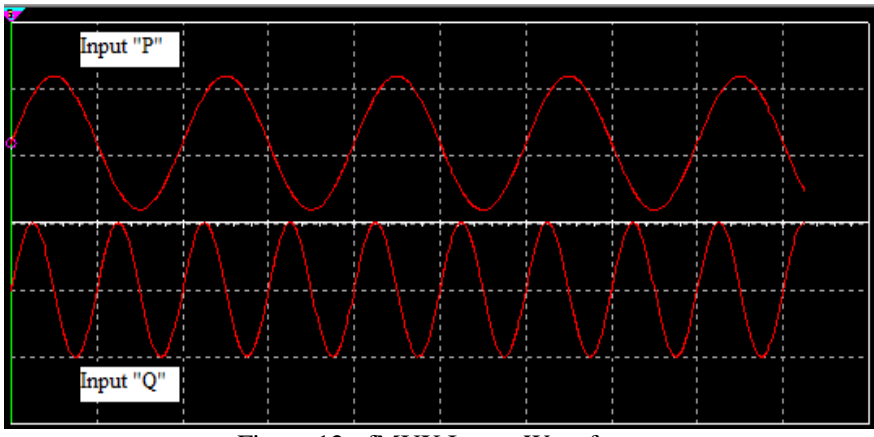

Figure 12:. fMUX Inputs Waveform 


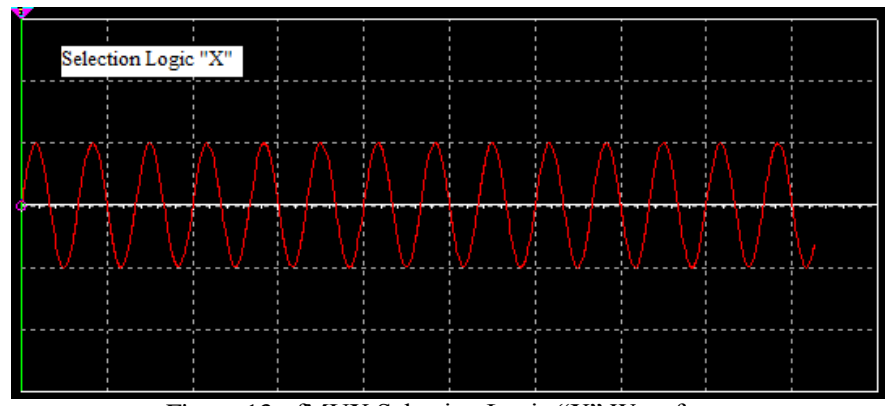

Figure 13:. fMUX Selection Logic " $\mathrm{X}$ " Waveform

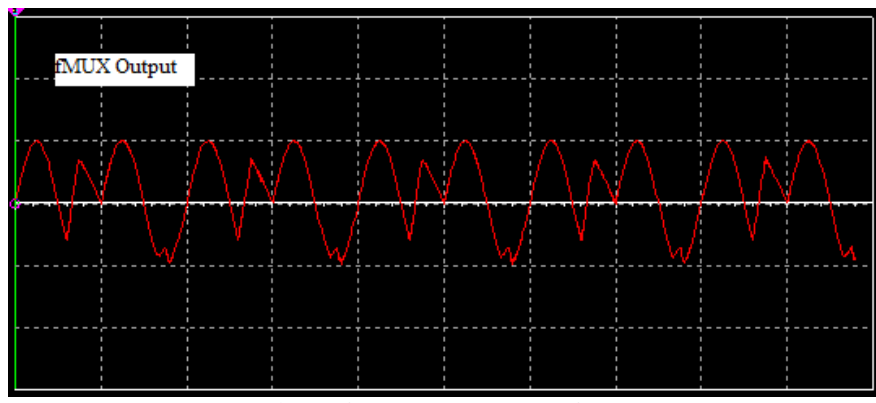

Fig 14 fMUX Output Waveform

\section{Generalization Of Fmux Using Digital Signal}

In the boundary conditions using the select variable " $\mathrm{X}$ ", we produce a binary switch (the same as being used in digital systems). It means that if $\mathrm{x}=1$, then output of "P" is taken. Likewise, if the value of $\mathrm{x}$ set to 0 leads to the output being equal to "Q" meaning that the value of "Q" is transferred to the output of the fMUX. The inputs selected for simulation of fMUX to show general digital behavior are Pulse Voltages of $\pm 1 \mathrm{~V}$.

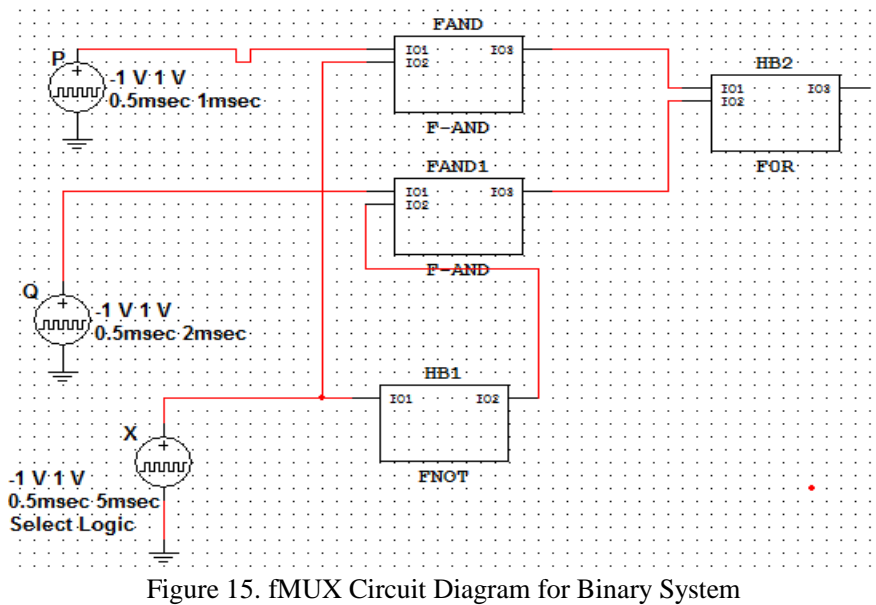

The simulation results were as follows in time base of $2 \mathrm{~ms} / \mathrm{Div}$ and voltage scale of 1V/Div.

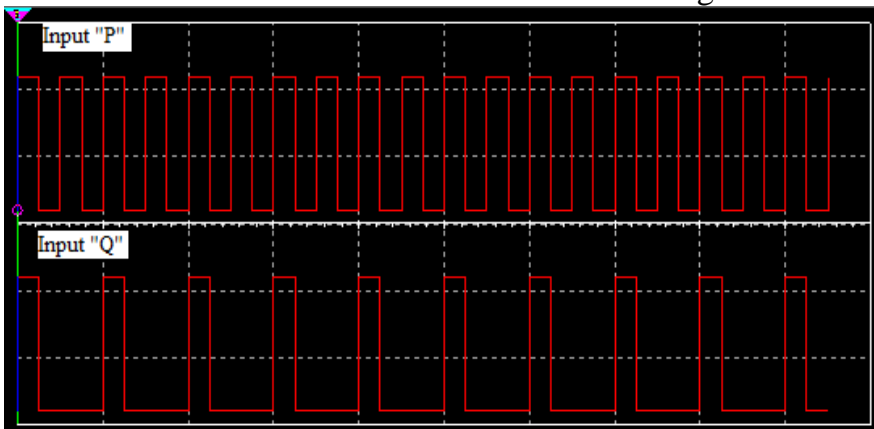

Figure 16 Inputs to fMUX 


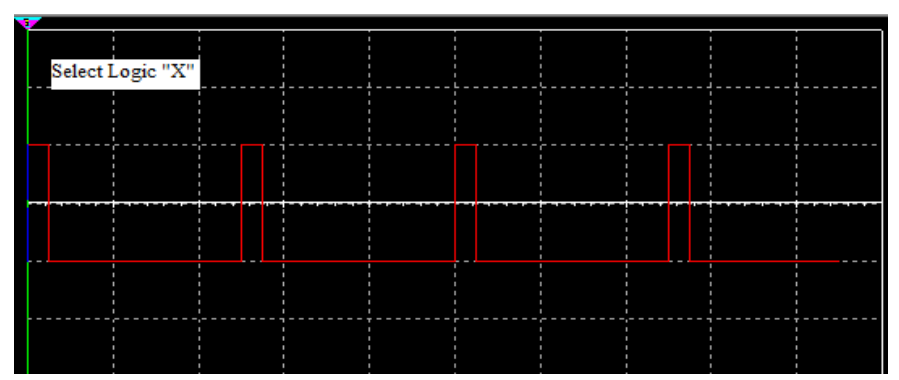

Figure 17:Select Logic "X” for fMUX

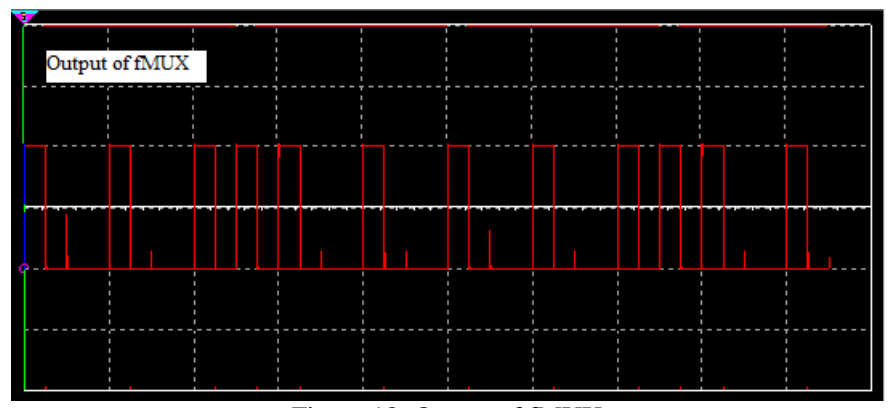

Figure 18: Output of fMUX

\section{Conclusions}

In this paper a design of Fuzzy Multiplexer was presented and its generalization to classical logic has been shown with simulations In this paper modified fuzzy logic gates where also introduced and there simulation was shown. These fuzzy gates were used to design fuzzy multiplexer or fMUX to perform multiplexing of two fuzzy signals with the logic of selection line and the corresponding simulations were shown. The fMUX was also tested for general binary signals and was found to be performing to desired standards.

fMUX introduced here could be used for the design of the fMUX networks for building fuzzy systems.

\section{References}

[1.] J. L. Pérez, M. A. Bañuelos, Electronic Models of Fuzzy Gates, Instrumentation and Development Vol. 3 Nr. 5, 1995.

[2.] Ben Choi, and K. Tipnis, New Components for Building Fuzzy Logic Circuits. FSKD, page 586-590. IEEE Computer Society, (2007)

[3.] Witold Pedrycz, Logic-driven fuzzy modeling with fuzzy multiplexers, Engineering Applications of Artificial Intelligence 17 (2004) 383-391

[4.] Antonio Gonzalez, Raul Perez, Completeness and consistency conditions for learning fuzzy rules, Fuzzy Sets and Systems 96 (1998) $37-51$

[5.] Tom Kazmierski, Fuzzy-logic digital-analogue interfaces for accurate mixed-signal simulation, 01/1998; In proceeding of: 1998 Design, Automation and Test in Europe (DATE '98), February 23-26, 1998, Le Palais des Congrès de Paris, Paris, France 